\title{
Gas separation in a Knudsen pump inspired by a Crookes radiometer
}

\author{
Tobias Baier $^{1} \cdot$ Steffen Hardt ${ }^{1}$
}

Received: 11 December 2019 / Accepted: 11 April 2020 / Published online: 6 May 2020

(c) The Author(s) 2020

\begin{abstract}
In a Knudsen pump, gas flow is induced by thermal gradients along a channel when the mean free path of the gas molecules is comparable to the geometric feature size. By periodically varying both the channel dimension and the reflection properties of gas molecules at the channel walls, a gas flow along the channel can be induced by application of a constant temperature difference between the channel walls. Inspired by the Crookes-Radiometer, one such arrangement consists of placing an array of plates with different reflection properties on their opposite sides along a channel. We investigate the transport of binary gas mixtures along such channels by direct simulation Monte Carlo (DSMC), focusing on the discrimination in transport of individual species due to gradients in temperature, composition and pressure. An exemplary separation column is investigated where a counterflow involving a combination of thermally induced, pressure driven and diffusion flows is established, resulting in an enrichment of the individual species at opposite ends of the column.
\end{abstract}

Keywords Rarefied gas dynamics · Knudsen pump · Gas separation · Direct Simulation Monte Carlo (DSMC)

\section{Introduction}

The Crookes radiometer (Crookes 1876) has fascinated physicists since its inception in 1874 and has played a pivotal role in advancing the understanding of gas dynamics (Reynolds 1879; Maxwell 1879). Recently, we investigated a Knudsen pump inspired by the Crookes radiometer (Baier et al. 2017), where a uniform array of plates are stacked along a channel between two parallel plates, as sketched in Fig. 1a. When the reflection properties for gas molecules on opposite sides of the plates (also referred to as vanes) are different - in our model system one is ideally specularly and the other is purely diffusely reflective at temperature $T_{1}-\mathrm{a}$ periodic temperature profile is induced along the channel when its sidewalls are kept at a different temperature $T_{2}$. At intermediate Knudsen numbers, when the mean free path $\ell$ of gas molecules is of the order of or smaller than the extension of a vane, the temperature variation around the tip of the vane induces a flow reminiscent of thermal edge flow (Sone and Yoshimoto 1997), predominantly driving the gas towards the side with higher temperature (Baier et al. 2017).

Tobias Baier

baier@nmf.tu-darmstadt.de

1 Institute for Nano- and Microfluidics, Technische Universität Darmstadt, 64287 Darmstadt, Germany
Thus, while in a Crookes radiometer the vanes are allowed to rotate on a spindle, the array of plates considered in the model system drive a flow along the channel.

As a Knudsen pump does not possess any moving parts, it lends itself for operation in low-maintenance situations such as for feeding gases to sensor surfaces. When the mixtures of gases are transported, it is of interest whether the pump may, adversely or favorably, influence the overall detection characteristic of such a combined device. Similarly, the separation of gas species by mass or size plays an important role for many analytical applications and in chemical engineering. In this paper, we thus investigate the transport of gas mixtures in the described Knudsen pump due to gradients in temperature, pressure and composition with special focus on species separation.

Isothermal diffusion in multicomponent gas mixtures was studied from the 1830s onwards by Thomas Graham in a series of seminal studies (Graham 1833, 1846, 1863; Mason and Evans 1969; Mason and Wright 1971) resulting in what are now known as Graham's laws of diffusion and effusion. According to these, it is possible to achieve gas separation by mass when passing gas mixtures through pores or small holes. While thermal diffusion in liquids was observed around the same time (Ludwig 1856; Soret 1879), thermal diffusion in gaseous mixtures was investigated theoretically much later by Enskog (1911) and Chapman (1918), with 


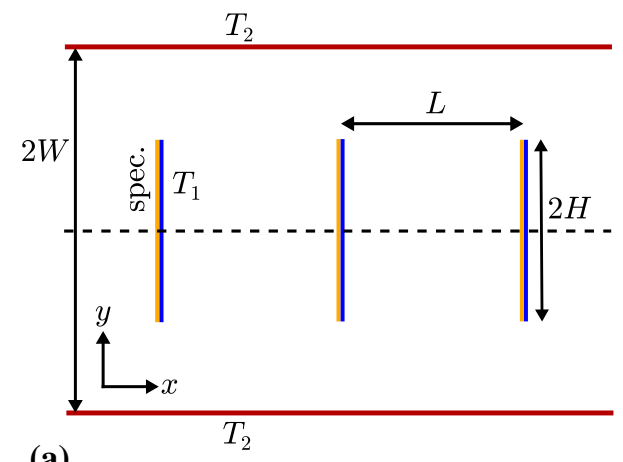

(a)

Fig. 1 a Sketch of the geometry: vanes with a specularly and a diffusely reflecting sides are stacked along a channel formed by parallel plates. b Thermally induced flow is simulated in one unit segment of the channel, utilizing the reflection symmetry at the channel centerplane and employing periodic boundary conditions at the left and

experimental validation shortly after (Chapman and Dootson 1917). It found practical application in the separation column described by Clusius and Dickel (1938); Grodzka and Facemire (1977), where thermal diffusion is coupled to a countercurrent flow in the gap between opposing hot and cold walls driven by natural convection. This scheme leads to an accumulation of the heavy and light components of the gas at the bottom and top of the column, respectively. A similar coupling between convection and diffusion is also expected in the proposed Knudsen pump, since it relies on a thermal gradient across the channel. However, while the Clusius-Dickel column relies on natural convection for driving an axial flow along the column, the system considered here is driven by thermally induced flow.

From the theory of non-equilibrium thermodynamics (de Groot and Mazur 1984; Fitts 1962) it is well known that for a system close to equilibrium the mass fluxes of mixture components together with the heat flux are intricately coupled to variations in pressure, composition and temperature. In the framework of the linearized Boltzmann equation, this was extensively studied and extended to gases at arbitrary rarefaction, thus situations in which the system is not even close to local equilibrium (Bishaev and Rykov 1984; Sharipov 1994a; Takata 2009; Sharipov 1994b; Sharipov and Kalempa 2002). For multicomponent gas flow along channels of various cross sections the cross-coupling of fluxes was investigated in Naris et al. (2004); Sharipov and Kalempa (2006); Kosuge and Takata (2008) and has found applications in the study of gas separation in Knudsen pumps. In that context, different channel cross sections and temperature profiles along their walls were studied (Takata et al. 2007; Takata and Umetsu 2011; Zhang et al. 2019). Furthermore, gas separation by thermal transpiration coupled to pressure driven flow across membranes was studied by Sugimoto and Hibino (2012); Nakaye and Sugimoto (b)

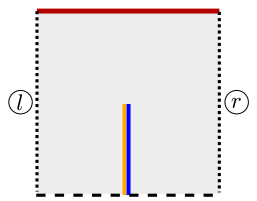

(c)

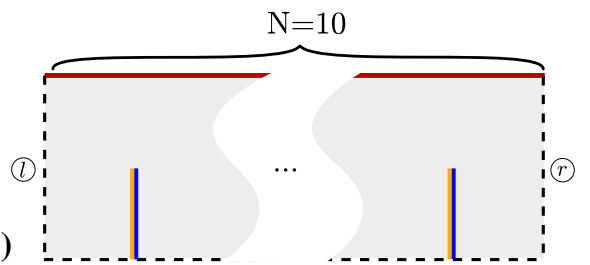

right boundaries. c Flows with pressure- and concentration gradients along the channel are simulated in a domain consisting of $N=10$ unit segments with appropriate pressure, concentration or reflection boundary conditions at the left and right boundaries

(2014); Nakaye et al. (2015); Nakaye and Sugimoto (2016); Matsumoto et al. (2016); Kosyanchuk et al. (2017); Tamura et al. (2019). Gas separation in Knudsen pumps based on thermal edge flow (Sugimoto and Sone 2005) was investigated in Sugimoto et al. (2007); Sugimoto (2008). Separation by transpiration through an array of pairs of closely spaced wire meshes at different temperature (Sugimoto et al. 2008) was studied in Yakunchikov and Kosyanchuk (2019a, b). All these systems bear some resemblance to the Knudsen pump of the present paper, but differ significantly in their geometric configuration and the method by which the thermal gradient driving the flow is created.

The organization of this paper is as follows: in Sect. 2 we describe the numerical model used and the method for evaluating the species and heat fluxes together with the corresponding transport coefficients. These are evaluated numerically in Sect. 3, first for purely thermally induced flow and subsequently including fluxes due to pressure and concentration gradients. In Sect. 4, a counterflow separation column utilizing a combination of the complementing transport mechanisms is proposed and investigated, followed by conclusions and an outlook.

\section{Methods}

Geometry The Knudsen pump under consideration consists of a periodic array of vanes of width $2 \mathrm{H}$ placed at separation $L$ in a channel formed by parallel plates at separation $2 W$ as sketched in Fig. 1a. We effectively assume a 2D situation with translational invariance normal to the $x y$-plane and will consider $W=L=2 H$ throughout. The walls of the channel are assumed to be diffusively reflecting at temperature $T_{2}$. The vanes have one diffusively reflecting side at temperature $T_{1}$, while their other side is assumed to be ideally specularly 
reflective. Thermally induced flow is activated by setting the wall temperatures to $T_{2}=2 T_{0}$ and $T_{1}=T_{0}$, with $T_{0}=300 \mathrm{~K}$ as reference temperature. During isothermal flow conditions due to a pressure or concentration gradient along the channel, $T_{2}=T_{1}=T_{0}$ applies on all diffusely reflecting walls.

For purely thermally induced flow the velocity, temperature and concentration fields are spatially periodic with the unit cell size. Therefore, together with the reflection symmetry at the channel center-plane it thus suffices to consider a unit segment of the channel around a single vane with periodic boundary conditions on its left and right boundaries, as shown in Fig. 1b. For flows with net pressure and concentration gradients along the channel, the simulations are performed in a domain consisting of $N=10$ unit segments with appropriate pressure, concentration or reflection boundary conditions at the left and right boundaries, see Fig. 1c.

DSMC simulations Simulations were performed using the direct simulation Monte Carlo (DSMC) method (Bird 1994) as implemented in the open-source fluid dynamics code OpenFoam (Weller et al. 1998; Scanlon et al. 2010), specifically release v1.0 of dsmcFOAM+ (White et al. 2018). Briefly, the DSMC method is a particle based method for solving the Boltzmann equation (Wagner 1992), with each DSMC particle representing a large number of physical atoms or molecules. The particle ensemble evolves by iterating the collisionless propagation along individual ballistic trajectories for a brief duration followed by an (instantaneous) collision step, where pairs of nearby particles (located in the same cell of a grid) are stochastically chosen to collide, with their pre- and post-collision momenta related according to a phenomenological model.

We focus on mixtures of Helium and Xenon modeled as variable hard spheres (VHS) with parameters given in Table 1 . The geometry is discretized by a uniform grid with a side-length of $a_{\text {cell }}=W / 100$ of one computational cell. The number of gas particles represented by a single DSMC particle was adjusted such that on average at least 40 DSMC particles are present in each cell for each species separately. The timestep $\Delta t$ for the simulation was chosen such that $\tilde{c} \Delta t<a_{\text {cell }} / 4$, where $\tilde{c}=\sqrt{2 k_{\mathrm{B}} T / m}$ is the characteristic velocity based on the mass $m$ of the lightest particle at the highest temperature $T$ in the channel, and $k_{\mathrm{B}}=1.380649 \cdot 10^{-23} \mathrm{~J} / \mathrm{K}$ is Boltzmann's constant. In cases where a pressure and concentration boundary condition is applied, the implicit method

Table 1 Species mass, VHS diameter and viscosity index $\left(\mu \sim T^{\omega}\right)$ used in the simulation with reference temperature $T_{\text {ref }}=273 \mathrm{~K}$ (from Bird (1994), Tables A1, A2). For He-Xe collisions the arithmetic mean diameter and viscosity index of the collision partners was used

\begin{tabular}{llll}
\hline & $m / \mathrm{kg}$ & $d / \mathrm{m}$ & $\omega$ \\
\hline $\mathrm{He}$ & $6.65 \times 10^{-27}$ & $2.33 \times 10^{-10}$ & 0.66 \\
$\mathrm{Xe}$ & $218 . \times 10^{-27}$ & $5.74 \times 10^{-10}$ & 0.85 \\
\hline
\end{tabular}

proposed by Liou and Fang (2000) and improved by Wang and $\mathrm{Li}$ (2004) was used, taking into account the flux of molecules at thermal velocities during low speed gas flows on such boundaries.

Non-dimensionalisation The choice of a characteristic temperature $T_{0}=300 \mathrm{~K}$ specifies a characteristic velocity $c_{0}=\sqrt{2 k_{\mathrm{B}} T_{0} / m_{\mathrm{He}}}$ and relates a pressure scale $p_{0}=n_{0} k_{\mathrm{B}} T_{0}$ to a characteristic particle number density $n_{0}$ via the ideal gas law. The mean free path $\ell_{\mathrm{He}}=1 /\left(\sqrt{2} \pi d_{\mathrm{He}}^{2} n_{0}\right)$ in a pure Helium atmosphere defines a dimensionless Knudsen number $\mathrm{Kn}=\ell_{\mathrm{He}} / W$, characterizing the rarefaction of the flow. We take this relation between the characteristic particle number density, $n_{0}$, and the Knudsen number, $\mathrm{Kn}$, to also hold in case of gas mixtures, as it offers a unique relation between the two. This ensures that a given Knudsen number specifies the same characteristic pressure irrespective of gas composition.

All gas properties are encoded in the particle distribution functions $f_{i}(\mathbf{r}, \mathbf{c})$ for each species in the mixture, which is governed by the Boltzmann equation (Landau and Lifschitz 1983). In particular, the number densities $n_{i}(\mathbf{r})=\int f_{i}(\mathbf{r}, \mathbf{c}) \mathrm{d} \mathbf{c}$ of each species $i \in\{\mathrm{He}, \mathrm{Xe}\}$ and of the mixture, $n=\sum_{i} n_{i}$, specify the molar fractions $x_{i}=n_{i} / n$. Since $\sum_{i} x_{i}=1$, it suffices to specify $x_{\mathrm{He}}$ in the binary mixture.

With the velocity $\mathbf{u}_{i}=n_{i}^{-1} \int \mathbf{c}_{i} f_{i}(\mathbf{r}, \mathbf{c}) \mathrm{d} \mathbf{c}$ of each component, the corresponding species flux along the channel

$j_{i}=\int\left(n_{i} \mathbf{u}_{i} \cdot \mathbf{e}_{x}\right) \mathrm{d} A_{x}$,

is obtained by integrating over a full cross sectional area $A_{x}$ placed normal to the axis of the channel, with $\mathbf{e}_{x}$ being the unit vector pointing along this axis. Specifically, in the unit segment of Fig. 1b this cross section was placed half-way between the left boundary and the plate, while it was placed at the center of the multi-element domain of Fig. 1c. Nondimensionalised species fluxes are defined by

$J_{i}=j_{i} /\left(A_{x} n_{0} c_{0}\right)$.

Additionally, since we deal with a binary system, we define the alternative fluxes

$J_{P}=\left(x_{\mathrm{He}, 0} J_{\mathrm{He}}+x_{\mathrm{Xe}, 0} J_{\mathrm{Xe}}\right)$

and

$J_{C}=x_{\mathrm{He}, 0}\left(J_{\mathrm{He}}-J_{\mathrm{Xe}}\right)$,

characterizing an overall species flux and diffusive flux, respectively (Sharipov 1994b; Naris et al. 2004; Kosuge and Takata 2008). The characteristic molar fractions $x_{\mathrm{He}, 0}=x_{0}$ and $x_{\mathrm{Xe}, 0}=1-x_{0}$ used here are specified as initial conditions, as detailed below. In the following, we will mostly focus on the case of equimolar concentration, $x_{0}=0.5$. In 
this case, $J_{P}$ and $J_{C}$ directly correspond to half the sum and difference of the two molar fluxes of the individual species.

The local heat flux $\mathbf{q}=\sum_{i} \int\left(\frac{1}{2} m_{i} c^{2}\right) f_{i}(\mathbf{r}, \mathbf{c}) \mathrm{d} \mathbf{c}$ is integrated over the (upper) channel wall with surface area $A_{n}$ and unit normal $\mathbf{n}$ pointing into the channel to obtain the heat flux across the channel wall,

$j_{T}=\sum_{i} \int_{A_{y}}(\mathbf{q} \cdot \mathbf{n}) \mathrm{d} A_{n}$.

The corresponding non-dimensional heat flux is defined as

$J_{T}=j_{T} /\left(A_{n} p_{0} c_{0}\right)$.

In situations without translational symmetry in channel direction, such as pressure or concentration driven transport, a net heat flux in channel direction may influence the transport along the channel. However, since no thermal gradient is actively applied in channel direction, this heat flux is much smaller than the heat flux directly between the channel walls and the vanes and will be disregarded in the analysis of the results, only focusing on the transverse heat flux.

Transport coefficients Classical non-equilibrium thermodynamics (de Groot and Mazur 1984; Fitts 1962) establishes a linear relation $J_{\alpha}=L_{\alpha \beta} X_{\beta}$ between fluxes $J_{\alpha}$ and driving forces $X_{\beta}$ with a symmetric matrix of transport coefficients $L_{\alpha \beta}$ for systems close to equilibrium. With the temperature difference across the channel,

$X_{T}=\Delta T / T_{0}=\left(T_{2}-T_{1}\right) / T_{1}$,

and the pressure and concentration differences averaged over one unit element of length $L$,

$X_{P}=-L \overline{\partial_{x} p} / p_{0}$,

$X_{C}=-L \overline{\partial_{x} x_{\mathrm{He}}} / x_{\mathrm{He}, 0}$,

as driving forces, the corresponding fluxes are $J_{T}, J_{P}$ and $J_{C}$ introduced in Eqs. (3), (4) and (6) above (Sharipov 1994b; Naris et al. 2004; Kosuge and Takata 2008). The corresponding transport coefficients $L_{\alpha \beta}$ with $\alpha, \beta \in\{P, C, T\}$ are reported below. Note that we are not always close to equilibrium, in particular for thermally induced flow $X_{T}=\Delta T / T_{0}=1$. The transport coefficients should thus be considered as suitably normalized fluxes here. However, even up to this large temperature difference we verified that the thermally induced flux scales very nearly linearly with $\Delta T / T_{0}$. Nevertheless, departures from linearity may be expected during flow induced by a combination of thermodynamic forces due to the large temperature and composition variations across the channel.

\section{Transport coefficients: numerical evaluation}

\subsection{Thermally induced flow}

We start by considering purely thermally induced flow with $T_{2}-T_{1}=T_{1}=T_{0}$. This is modeled in the unit cell of Fig. $1 \mathrm{~b}$ with periodic boundary conditions on the left and right sides. Initial conditions in the channel are given by the density $n_{0}=n_{0}(\mathrm{Kn})$, gas composition $x_{\mathrm{He}}=x_{0}$ and temperature $T_{0}$. The difference in temperature in the gas on both sides of the tip of the vanes induces a flow across the vanes from the colder to the warmer side, as was extensively studied for a pure gas in Baier et al. (2017). Since the temperature close to the right-hand side of each vane is lower than the temperature close to its left-hand side, a flow from right to left is induced. Exemplary streamlines of the individual components for $x_{0}=0.5$ are shown in Figs. $2 \mathrm{a}$ and $3 \mathrm{a}$ for $\mathrm{Kn}=5.7$ and 0.57 , respectively. It is evident that both components follow similar flow patterns, although the heavier component has a disposition to follow straighter paths compared to the lighter component. Note that the heavier (and larger) component has a tendency to accumulate at the colder regions in the channel compared to the lighter (and smaller) component (Bird et al. 2007). Corresponding profiles of $x_{\mathrm{He}}$ and $n / n_{0}$ at $\mathrm{Kn}=5.7$ and 0.57 are shown in panel (b) and (c) of Figs. 2 and 3. Both of these different behaviors are expected to result in a difference in transport of the individual components of the mixture.

The transport coefficients $L_{\mathrm{PT}}$ and $L_{\mathrm{CT}}$ are shown in Fig. $4 \mathrm{a}$ for $x_{\mathrm{He}, 0}=0.5$. Note that since $X_{T}=1$, the species fluxes $J_{\alpha}$ and transport coefficients $L_{\alpha \mathrm{T}}$ are identical here. The transport coefficient $L_{\mathrm{PT}}$, corresponding to the total species flux, follows the same dependence on $\mathrm{Kn}$ as was observed for a single-component gas (Baier et al. 2017), with a maximum at some intermediate Knudsen number with a drop towards high and low Knudsen numbers. Essentially the same behavior is also observed for the transport coefficient $L_{\mathrm{CT}}$, corresponding to the difference in the fluxes of the individual components, with its maximum appearing at the same $\mathrm{Kn}$. This clearly indicates the possibility of separation (or discrimination in transport of mixture components) by thermally induced flow in the considered Knudsen pump, with the light component migrating faster than the heavier one.

To assess the dependence of the transport on composition, the ratio of the thermally induced species fluxes $J_{\mathrm{He}} / J_{\mathrm{Xe}}$ as function of the molar fraction $x_{0}=x_{\mathrm{He}, 0}$ is of interest. For simple convective transport at low Kn, one expects that $J_{i} \sim x_{i, 0}$. Therefore, we show in Fig. $4 \mathrm{~b}$ the ratio between the individual fluxes normalized by their 


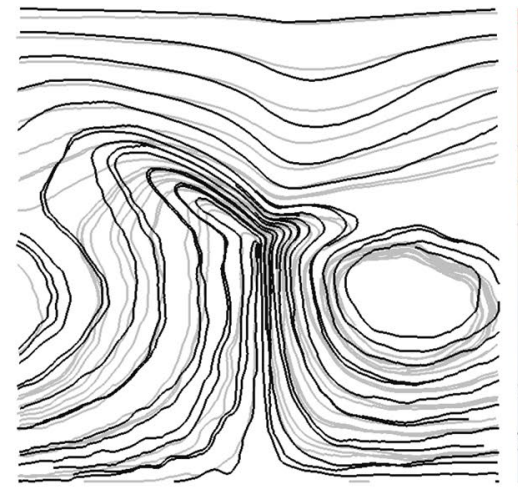

(a) Streamlines for He (black) and Xe (gray)

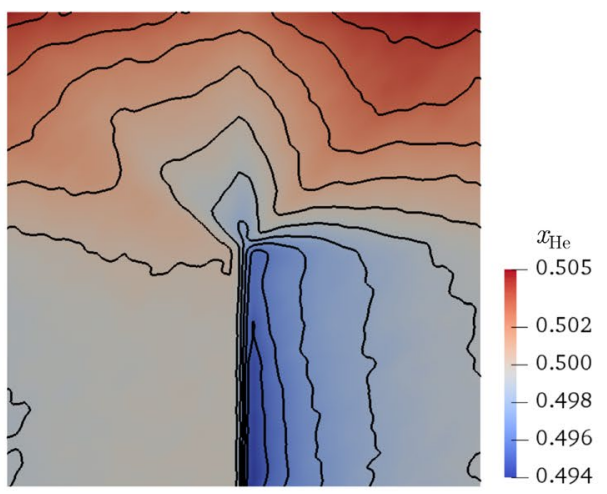

(b) Contours of $x_{\mathrm{He}}$

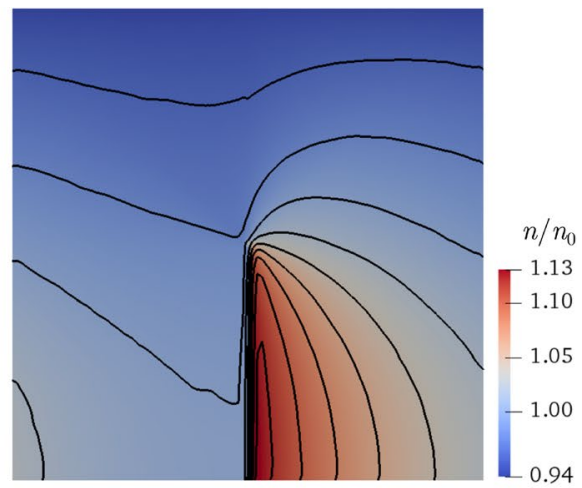

(c) Contours of $n / n_{0}$

Fig. 2 Streamlines, composition and number density during thermally induced flow of $\mathrm{He}$ and $\mathrm{Xe}$ at $\mathrm{Kn}=5.7, x_{0}=0.5$ and $\Delta T / T_{0}=1$. The flow direction is from right to left

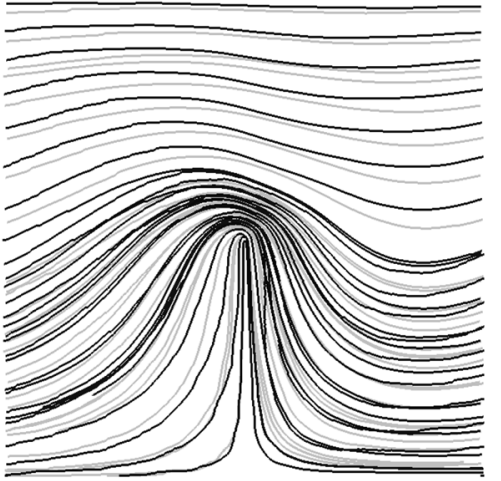

(a) Streamlines for He (black) and Xe (gray)

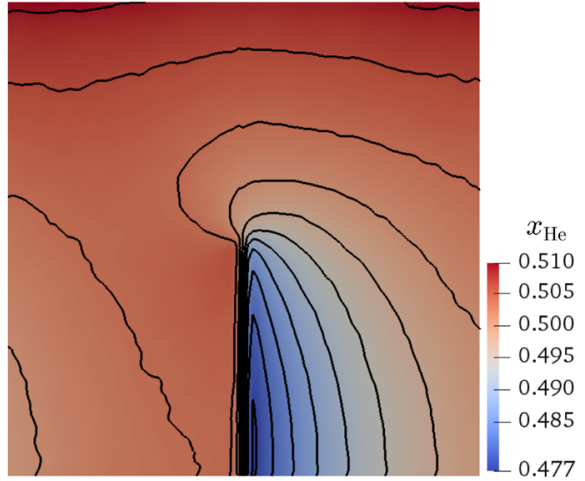

(b) Contours of $x_{\mathrm{He}}$

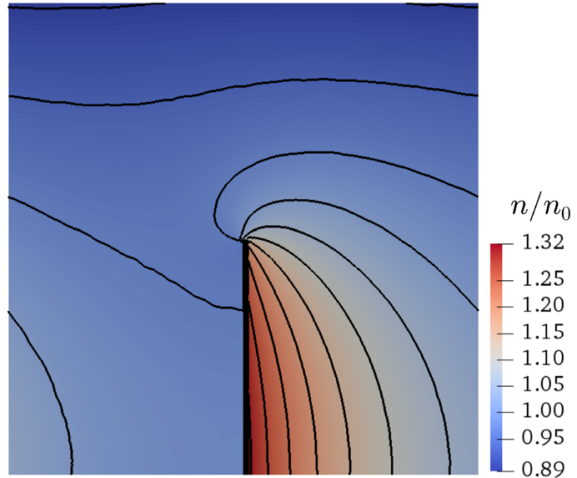

(c) Contours of $n / n_{0}$

Fig. 3 Streamlines, composition and number density during thermally induced flow of He and $\mathrm{Xe}$ at $\mathrm{Kn}=0.57, x_{0}=0.5$ and $\Delta T / T_{0}=1$. The flow direction is from right to left

corresponding molar fraction, $\left(J_{\mathrm{He}} / x_{\mathrm{He}, 0}\right) /\left(J_{\mathrm{Xe}} / x_{\mathrm{Xe}, 0}\right)$. The latter quantity is approximately the ratio of the average species velocities. For relatively small $\mathrm{Kn}=0.57$ the expectation is indeed fulfilled very well, with the ratio $\left(J_{\mathrm{He}} / x_{\mathrm{He}, 0}\right) /\left(J_{\mathrm{Xe}} / x_{\mathrm{Xe}, 0}\right)$ attaining a constant value. For larger $\mathrm{Kn}$ there is a noticeable dependence of the ratio of normalized fluxes on the composition, with a deviation of approximately $20 \%$ of the value at $x_{0}=0.5$ from the extremal values obtained for $\mathrm{Kn}=5.7$. Since this dependence is relatively weak in the region of interest, in what follows we will focus on the case $x_{0}=0.5$, representing an equal molar mixture of $\mathrm{He}$ and $\mathrm{Xe}$.

\subsection{Fluxes due to pressure and concentration gradients}

While the results of the previous section indicate that thermally induced flow can lead to species separation in the Knudsen pump considered, the temperature gradient across the channel will in general not be the only thermodynamic force inducing transport. In particular, since the separation goes hand in hand with a net mass transport, both a pressure gradient as well as a concentration gradient will in general develop along the axis of the pump when operated between two reservoirs or as a column with closed ends. 


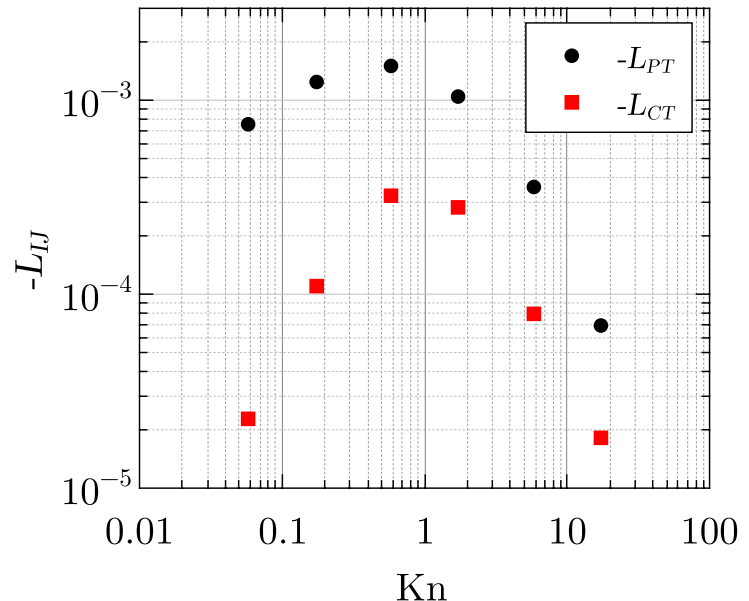

(a)

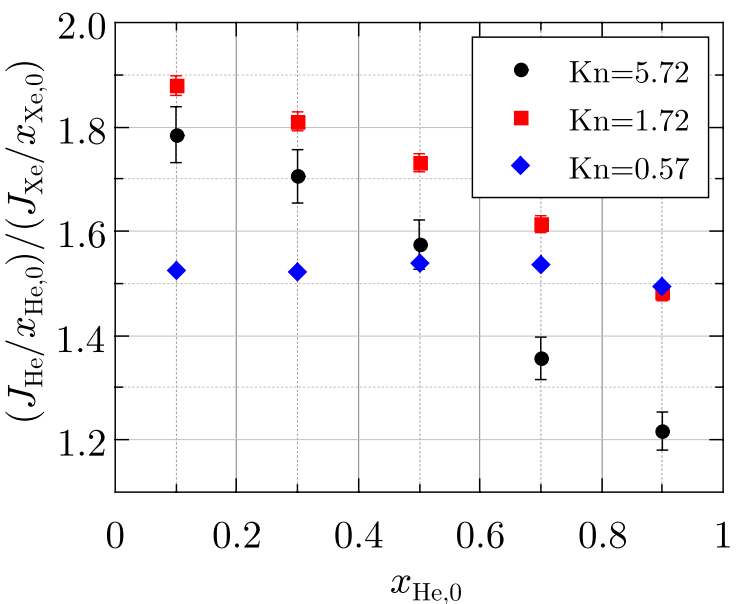

(b)

Fig. 4 Thermally induced transport. a Transport coefficients $-L_{\mathrm{PT}}$ and $-L_{\mathrm{CT}}$ for $x_{0}=0.5$. b Normalized flux ratio $\left(J_{\mathrm{He}} / x_{\mathrm{He}, 0}\right) /\left(J_{\mathrm{Xe}} / x_{\mathrm{Xe}, 0}\right)$ vs. composition $x_{0}=x_{\mathrm{He}, 0}$ at various Knudsen numbers

We, therefore, individually consider pressure and concentration driven flow by specifying pressures $1.3 p_{0}$ and $p_{0}$ or molar fractions $1.1 x_{0}$ and $0.9 x_{0}$ on the left and right sides of the channel, respectively. The equations are solved in a domain consisting of $N=10$ unit cells, see Fig. 1c. These values overwrite the default conditions $n_{0}=n_{0}(\mathrm{Kn}), x_{0}=0.5$ and $T_{0}=300 \mathrm{~K}$, which serve as initial conditions throughout the domain and as inlet and outlet boundary conditions unless otherwise specified. The temperatures $T_{1}$ and $T_{2}$ of the diffusely reflecting wall segments are kept at $T_{0}$. Species fluxes are monitored at the channel center until a steady state is reached (in which case they also become equal to

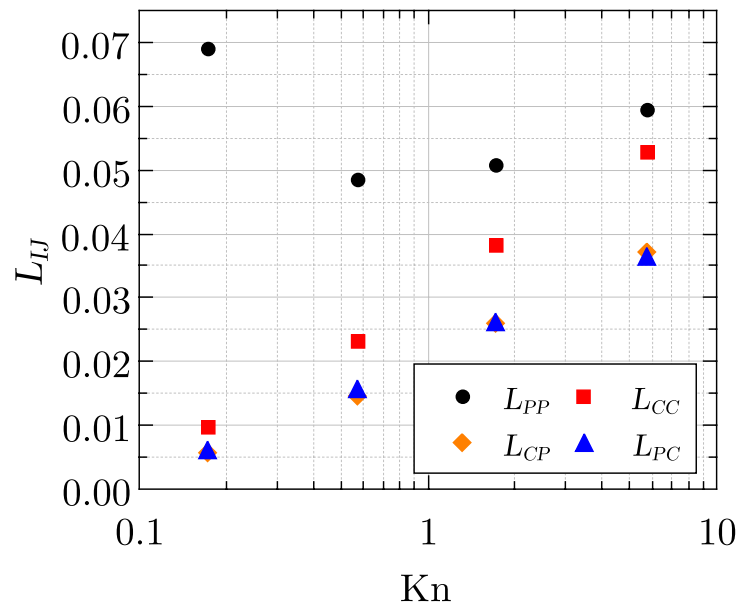

(a)

Fig. 5 a Transport coefficients $L_{\mathrm{PP}}, L_{\mathrm{CC}}$ and $L_{\mathrm{CP}} \approx L_{\mathrm{PC}}$ for concentration and pressure driven transport at $x_{0}=0.5$. b Transport coefficient $L_{\mathrm{TT}}$ for heat exchanged during thermally induced transport at the fluxes through the first element on the left hand side of the domain).

The corresponding transport coefficients $L_{\mathrm{PP}}, L_{\mathrm{CC}}, L_{\mathrm{CP}}$ and $L_{\mathrm{PC}}$ are shown in Fig. 5a. Just as for flow along a straight channel of constant cross-section, $L_{\mathrm{PP}}$ has a minimum at some intermediate $\mathrm{Kn}$ number. The transport coefficients $L_{\mathrm{CC}}, L_{\mathrm{CP}}$ and $L_{\mathrm{PC}}$ increase monotonically with $\mathrm{Kn}$, also familiar from straight channel flow (Naris et al. 2004). The Onsager reciprocal relation $L_{\mathrm{CP}}=L_{\mathrm{PC}}$, i.e., the symmetry of the matrix of transport coefficients, is fulfilled very well. It is evident that pressure-driven transport also contributes to $J_{C}$, permitting a separation of gas species.

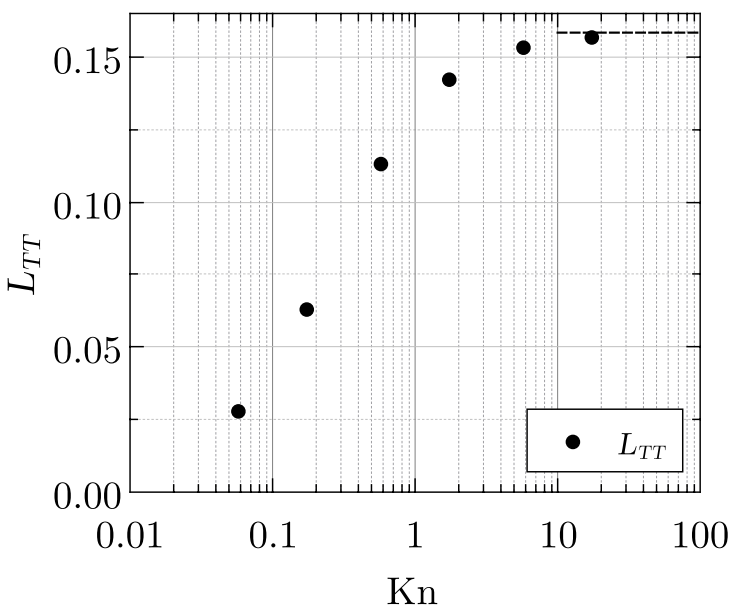

(b)

$x_{0}=0.5$. The dotted line corresponds to the value obtained numerically in the collisionless case 
The transport coefficient $L_{\mathrm{TT}}$ from the simulations of Sect. 3.1 is shown in Fig. 5b. Note that $L_{\mathrm{TT}}$ increases linearly with $\mathrm{Kn}$ in the continuum regime (where heat conduction dominates over convective transport of heat) and saturates towards the collisionless regime of large Kn. Not too far from equilibrium, the transport coefficients $L_{\mathrm{TP}}$ and $L_{\mathrm{TC}}$ are expected to be equal to the corresponding coefficients $L_{\mathrm{PT}}$ and $L_{\mathrm{CT}}$ shown in Fig. 4 a by Onsager's reciprocal relations $L_{\alpha \beta}=L_{\beta \alpha}$. However, while the individual mass fluxes due to pressure and concentration gradients are large, the corresponding heat fluxes are very small, resulting in large errors for the corresponding transport coefficients in our stochastic simulations. Since these transport coefficients are not used in the following, we do not presently expend the necessary integration times needed in to obtain reliable values.

\section{Separation column}

As an example of a separation column we consider a series of $N=10$ unit elements as in the previous section, but assume the system to be closed by specularly reflecting endcaps on the left and right (which here merely serve as a convenient way to close the domain). The primary driving force is again a temperature difference between the channel walls at temperature $T_{2}=2 T_{0}$ and the vanes at temperature $T_{1}=T_{0}$. The simulations were again started with initial conditions $n_{0}=n_{0}(\mathrm{Kn}), x_{0}=0.5$ and $T_{0}=300 \mathrm{~K}$ throughout the

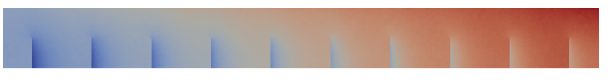

(a) $x_{\mathrm{He}}$ at $\mathrm{Kn}=0.57$

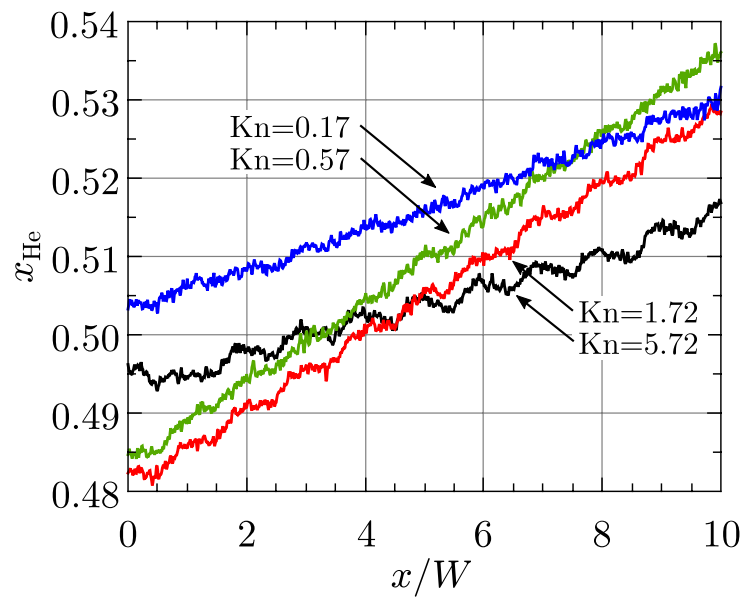

(c)

Fig. 6 Composition, $x=x_{\mathrm{He}}$ in $\mathbf{a}$, $\mathbf{c}$, and pressure, $p / p_{0}$ in $\mathbf{b}$, $\mathbf{d}$, along the channel with $N=10$ elements and symmetry endcaps. The color scale in the 2D plots in $\mathbf{a}$ and $\mathbf{b}$ showing exemplary composition and domain and were run until the net particle flux measured at the center of the domain becomes vanishingly small.

The simulated pressure and molar fraction of $\mathrm{He}$ along the wall of the separation column are shown in Fig. 6. Note that $x=x_{\mathrm{He}}$ is larger on the right. While pure thermally induced transport preferentially pumps $\mathrm{He}$ to the left, the pressuredriven counter flow dominates, preferentially pumping He to the right. Aside from slight perturbations within the first two unit cells adjacent to the endcaps, both sets of curves show a constant slope on average, with slight undulations due to the discrete periodicity along the channel. The corresponding driving forces $X_{P}$ and $X_{C}$ averaged over the central six unit cells are shown in Table 2.

In equilibrium the net species flows $J_{i}$ along the channel vanishes. Assuming linear transport and solving $J_{P}=L_{P \beta} X_{\beta}=0$ and $J_{C}=L_{C \beta} X_{\beta}=0$ at prescribed $X_{T}$ yields

Table 2 Pressure and concentration gradients in the separation column, evaluated by direct numerical simulation and by assuming linear transport via Eq. (10)

\begin{tabular}{llllll}
\hline \multirow{2}{*}{ Kn } & \multicolumn{2}{l}{ Numerical, Fig. 6 } & & \multicolumn{2}{l}{ Lin. transport, Eq. (10) } \\
\cline { 2 - 3 } \cline { 5 - 6 } & $X_{P} / X_{T}$ & $X_{C} / X_{T}$ & & $X_{P} / X_{T}$ & $X_{C} / X_{T}$ \\
\hline 0.17 & $3.0 \times 10^{-2}$ & $-2.7 \times 10^{-3}$ & & $1.8 \times 10^{-2}$ & $+0.8 \times 10^{-3}$ \\
0.57 & $4.7 \times 10^{-2}$ & $-5.3 \times 10^{-3}$ & & $3.4 \times 10^{-2}$ & $-7.5 \times 10^{-3}$ \\
1.72 & $3.5 \times 10^{-2}$ & $-4.8 \times 10^{-3}$ & & $2.6 \times 10^{-2}$ & $-10 \times 10^{-3}$ \\
5.72 & $1.2 \times 10^{-2}$ & $-2.1 \times 10^{-3}$ & & $0.9 \times 10^{-2}$ & $-4.8 \times 10^{-3}$ \\
\hline
\end{tabular}

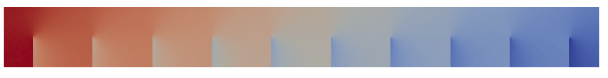

(b) $p / p_{0}$ at $\mathrm{Kn}=0.57$

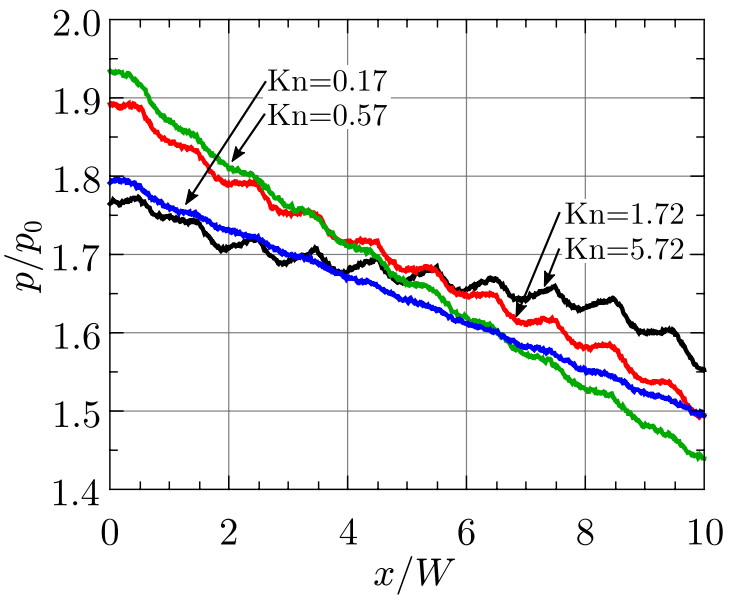

(d)

pressure profiles in the geometry for $\mathrm{Kn}=0.57$ can be inferred from c and $\mathbf{d}$, where the corresponding profiles along the sidewalls of the channel are shown 
$\frac{X_{P}}{X_{T}}=\frac{L_{\mathrm{PC}} L_{\mathrm{CT}}-L_{\mathrm{PT}} L_{\mathrm{CC}}}{L_{\mathrm{PP}} L_{\mathrm{CC}}-L_{\mathrm{PC}} L_{\mathrm{CP}}} \quad$ and $\quad \frac{X_{C}}{X_{T}}=\frac{L_{\mathrm{CP}} L_{\mathrm{PT}}-L_{\mathrm{CT}} L_{\mathrm{PP}}}{L_{\mathrm{PP}} L_{\mathrm{CC}}-L_{\mathrm{PC}} L_{\mathrm{CP}}}$

A comparison between pressure and concentration gradients obtained numerically in the separation column and assuming linear transport is given in Table 2. While the pressure gradient is predicted about $40 \%$ too low by the linear theory, the predicted concentration gradient is nearly twice as large compared to the direct simulation for $\mathrm{Kn}>0.5$ and is even predicted to change sign for lower $\mathrm{Kn}$. This indicates that the linear relationship between driving forces and fluxes is only approximately fulfilled during transport under combined thermodynamic forces, despite the nearly linear increase of $J_{i}$ with the applied temperature difference $\Delta T / T_{0}$ during thermally induced transport alone. Generally, for such a scenario with coupled fluxes and a temperature interval as large as $\left[T_{0}, 2 T_{0}\right]$, one does not necessarily expect the linear theory to provide a good approximation. Nevertheless, both schemes agree that an optimum of separation can be obtained at Kn close to unity.

\section{Conclusions and outlook}

The transport properties of a Helium-Xenon gas mixture in a Knudsen pump inspired by the Crookes radiometer were investigated numerically with particular attention to gas separation. The Knudsen pump consists of a channel with vanes possessing one specularly and one diffusely reflecting side. Temperature $(T)$, pressure $(P)$ and concentration $(C)$ gradients were considered as driving forces. It is found that the transport coefficients (or suitably normalized fluxes) $L_{\mathrm{PT}}, L_{\mathrm{CT}}$ and $L_{\mathrm{PP}}$ possess extrema at Kn close to unity, while all other transport coefficients increase monotonicaly with $\mathrm{Kn}$. In an exemplary separation column based on opposing thermally induced and pressure-driven fluxes, gas separation is achieved with an optimum performance for Kn close to unity. In situations when the Knudsen pump is used for feeding gas mixtures to sensor surfaces, the preferential transport of different gas components may influence the detection characteristics of the sensor system.

In our simulations the size of the separation column was limited to a relatively small number of unit cells by the computational cost of the DSMC method. Nevertheless, an enrichment of Helium and Xenon of several percent was found on the opposing ends of this short column. It is thus expected that a substantially longer column will be able to effectively separate two gas species. This can be further improved by connecting several such columns in series. Indeed, a considerably longer column will likely be necessary when separating gas mixtures composed of species with a smaller difference in mass than considered here.
As indicated in the introduction, several Knudsen pumps have been investigated that all have a substantial potential for separating gas mixtures. At the moment data are too sparse to favor one over the other. In particular, the separation may strongly depend on details of the wall-molecule interaction. This also applies to the model investigated by us, as purely specularly reflective walls clearly are an idealization. Departures from the ideal case were investigated in Baier et al. (2017) for the mass flux of a single-component gas. A more realistic model would not only have to incorporate realistic models for the accommodation coefficients at the walls, it would also need to account for the differences in wall interactions of different gas species. Our focus on the ideal case should therefore be viewed as a prototypic scenario relying on a thermal gradient across two sides of a vane for inducing the flow of gas. A similar effect on species transport is expected when the opposite sides of the vanes have different temperatures due to preferential heating or cooling.

Acknowledgements Open Access funding provided by Projekt DEAL. Financial support by the DFG (Deutsche Forschungsgemeinschaft) under grant number HA 2696/41-1 is gratefully acknowledged.

Open Access This article is licensed under a Creative Commons Attribution 4.0 International License, which permits use, sharing, adaptation, distribution and reproduction in any medium or format, as long as you give appropriate credit to the original author(s) and the source, provide a link to the Creative Commons licence, and indicate if changes were made. The images or other third party material in this article are included in the article's Creative Commons licence, unless indicated otherwise in a credit line to the material. If material is not included in the article's Creative Commons licence and your intended use is not permitted by statutory regulation or exceeds the permitted use, you will need to obtain permission directly from the copyright holder. To view a copy of this licence, visit http://creativecommons.org/licenses/by/4.0/.

\section{References}

Baier T, Hardt S, Shahabi V, Roohi E (2017) Knudsen pump inspired by Crookes radiometer with a specular wall. Phys Rev Fluids 2(3):033401. https://doi.org/10.1103/PhysRevFluids.2.033401

Bird GA (1994) Molecular gas dynamics and the direct simulation of gas flows. Clarendon Press, Oxford

Bird RB, Stewart WE, Lightfoot EN (2007) Transport phenomena. Wiley, Hoboken

Bishaev AM, Rykov VA (1984) The symmetry of the Onsager kinetic coefficients in a rarefied gas. Fluid Dyn 19(6):950-955. https:// doi.org/10.1007/BF01411585

Chapman S (1918) On the kinetic theory of a gas. Part II. Philos Trans R Soc Lond A 217(549-560):115-197. https://doi.org/10.1098/ rsta.1918.0005

Chapman S, Dootson F (1917) A note on thermal diffusion. Lond Edinb Dublin Philos Mag J Sci 33(195):248-253. https://doi. org/10.1080/14786440308635635

Clusius K, Dickel G (1938) Neues Verfahren zur Gasentmischung und Isotopentrennung. Naturwissenschaften 26(33):546-546. https:// doi.org/10.1007/BF01675498 
Crookes W (1876) Improvement in apparatus for indicating the intensity of radiation. US patent 182172

de Groot SR, Mazur P (1984) Non-equilibrium thermodynamics. Dover Publications, Washington

Enskog D (1911) A generalization of Maxwell's second kinetic gas theory. Physik Z 12:56-68

Fitts DD (1962) Nonequilibrium thermodynamics. McGraw-Hill, New York

Graham T (1833) On the law of the diffusion of gases. Philos Mag 2:175. https://doi.org/10.1080/14786443308648004

Graham T (1846) On the motion of gases. Philos Trans R Soc Lond 136:573-631. https://doi.org/10.1098/rstl.1846.0029

Graham T (1863) XVII. On the molecular mobility of gases. Philos Trans R Soc Lond 153:385-405. https://doi.org/10.1098/ rstl.1863.0017

Grodzka PG, Facemire B (1977) Clusius-Dickel separation: a new look at an old technique. Sep Sci 12(2):103-169

Kosuge S, Takata S (2008) Database for flows of binary gas mixtures through a plane microchannel. Eur J Mech B Fluids 27(4):444465. https://doi.org/10.1016/j.euromechflu.2007.08.002

Kosyanchuk V, Kovalev V, Yakunchikov A (2017) Multiscale modeling of a gas separation device based on effect of thermal transpiration in the membrane. Sep Purif Technol 180:58-68. https://doi. org/10.1016/j.seppur.2017.02.038

Landau LD, Lifschitz EM (1983) Physikalische Kinetik. AkademieVerlag, Berlin

Liou W W, Fang Y C (2000) Implicit boundary conditions for direct simulation Monte Carlo method in MEMS flow predictions. Comput Model Eng Sci 1(4):119-128. https://doi.org/10.3970/ cmes.2000.001.571

Ludwig C (1856) Diffusion zwischen ungleich erwärmten Orten gleich zusammengesetzter Lösung. Sitzungsbericht Kaiser Akad Wiss 65:539

Mason EA, Evans RB (1969) Graham's laws: simple demonstrations of gases in motion: part I. Theory J Chem Educ 46(6):358. https ://doi.org/10.1021/ed046p358

Mason EA, Wright PG (1971) Graham's laws. Contemp Phys 12(2):179-186

Matsumoto M, Nakaye S, Sugimoto H (2016) Gas separation by the molecular exchange flow through micropores of the membrane. AIP Conf Proc 1786:080011. https://doi.org/10.1063/1.4967604

Maxwell JC (1879) On stresses in rarified gases arising from inequalities of temperature. Philos Trans R Soc Lond 170:231-256. https ://doi.org/10.1098/rstl.1879.0067

Nakaye S, Sugimoto H (2014) Numerical analysis of gas separator with thermal transpiration in micro channels II. AIP Conf Proc 1628:792-798. https://doi.org/10.1063/1.4902674

Nakaye S, Sugimoto H (2016) Demonstration of a gas separator composed of Knudsen pumps. Vacuum 125:154-164. https://doi. org/10.1016/j.vacuum.2015.12.015

Nakaye S, Sugimoto H, Gupta NK, Gianchandani YB (2015) Thermally enhanced membrane gas separation. Eur J Mech B Fluids 49:36-49. https://doi.org/10.1016/j.euromechflu.2014.07.004

Naris S, Valougeorgis D, Kalempa D, Sharipov F (2004) Gaseous mixture flow between two parallel plates in the whole range of the gas rarefaction. Phys A 336(3-4):294-318. https://doi.org/10.1016/j. physa.2003.12.047

Reynolds O (1879) On certain dimensional properties of matter in the gaseous state. Philos Trans R Soc Lond 170:727-845. https://doi. org/10.1098/rstl.1879.0078

Scanlon TJ, Roohi E, White C, Darbandi M, Reese JM (2010) An open source, parallel DSMC code for rarefied gas flows in arbitrary geometries. Comput Fluids 39(10):2078-2089. https://doi. org/10.1016/j.compfluid.2010.07.014

Sharipov F (1994) Onsager-Casimir reciprocity relations for open gaseous systems at arbitrary rarefaction: I. General theory for single gas. Phys A 203(3-4):437-456. https://doi.org/10.1016/03784371(94)90009-4

Sharipov F (1994) Onsager-Casimir reciprocity relations for open gaseous systems at arbitrary rarefaction III. Theory and its application for gaseous mixtures. Phys A 209(3-4):457-476. https://doi. org/10.1016/0378-4371(94)90197-X

Sharipov F, Kalempa D (2002) Gaseous mixture flow through a long tube at arbitrary Knudsen numbers. J Vac Sci Technol A 20(3):814-822. https://doi.org/10.1116/1.1469010

Sharipov F, Kalempa D (2006) Onsager-Casimir reciprocal relations based on the Boltzmann equation and gas-surface interaction. Gaseous mixtures. J Stat Phys 125(3):661-675. https://doi. org/10.1007/s10955-006-9200-1

Sone Y, Yoshimoto M (1997) Demonstration of a rarefied gas flow induced near the edge of a uniformly heated plate. Phys Fluids 9(11):3530-3534. https://doi.org/10.1063/1.869461

Soret C (1879) Sur l'etat d'équilibre que prend au point de vue de sa concentration une dissolution saline primitivement homogène dont deux parties sont portées a des températures difféntes. Arch Sci Phys Nat Genève 2:48-61

Sugimoto H (2008) Experiment on the gas separation effect of the pump driven by the thermal edge flow. AIP Conf Proc 1084(1):1123-1128. https://doi.org/10.1063/1.3076450

Sugimoto H, Hibino M (2012) Numerical analysis on gas separator with thermal transpiration in micro channels. AIP Conf Proc 1501:794-801. https://doi.org/10.1063/1.4769623

Sugimoto H, Sone Y (2005) Vacuum pump without a moving part driven by thermal edge flow. In Rarefied Gas Dynamics: 24th International symposium on rarefied gas dynamics RGD 24, vol 762, pp 168-173. https://doi.org/10.1063/1.1941531

Sugimoto H, Takata S, Kosuge S (2007) Gas separation effect of the pump driven by the thermal edge flow. In: Ivanov MK, Rebrov AK (eds) Rarefied gas dynamics. Siberian Branch of the Russian Academy of Sciences, Novosibirsk, pp 1158-1163

Sugimoto H, Kawakami S, Moriuchi K (2008) Rarefied gas flows induced through a pair of parallel meshes with different temperatures. AIP Conf Proc 1084:1021-1026. https://doi. org/10.1063/1.3076432

Takata S (2009) Symmetry of the linearized Boltzmann equation II. J Stat Phys 136(5):945-983. https://doi.org/10.1007/s1095 5-009-9805-2

Takata S, Umetsu H (2011) Numerical study on effective configurations of the Knudsen pump for separation and compression. AIP Conf Proc 1333:998-1003. https://doi.org/10.1063/1.3562776

Takata S, Sugimoto H, Kosuge S (2007) Gas separation by means of the Knudsen compressor. Eur J Mech B Fluids 26(2):155-181. https://doi.org/10.1016/j.euromechflu.2006.05.002

Tamura S, Sugimoto H, Yashima M (2019) Desktop gas isotope separation by Knudsen pump. AIP Conf Proc 2132(1):190007. https:// doi.org/10.1063/1.5119679

Wagner W (1992) A convergence proof for bird's direct simulation monte carlo method for the boltzmann equation. J Stat Phys 66(3-4):1011-1044

Wang M, Li Z (2004) Simulations for gas flows in microgeometries using the direct simulation Monte Carlo method. Int J Heat Fluid Flow 25(6):975-985. https://doi.org/10.1016/j.ijheatfluidflow .2004.02.024

Weller HG, Tabor G, Jasak H, Fureby C (1998) A tensorial approach to computational continuum mechanics using object-oriented techniques. Comput Phys 12(6):620-631. https://doi. org/10.1063/1.168744

White C, Borg MK, Scanlon TJ, Longshaw SM, John B, Emerson DR, Reese JM (2018) dsmcFoam+: an openFOAM based direct simulation Monte Carlo solver. Comput Phys Commun 224:22-43. https://doi.org/10.1016/j.cpc.2017.09.030 
Yakunchikov A, Kosyanchuk V (2019a) Numerical investigation of gas separation in the system of filaments with different temperatures. Int J Heat Mass Transfer 138:144-151. https://doi.org/10.1016/j. ijheatmasstransfer.2019.04.020

Yakunchikov A, Kosyanchuk V (2019b) Gas separation effect induced by filaments with different temperatures. AIP Conf Proc 2132(1):180003. https://doi.org/10.1063/1.5119661

Zhang Z, Wang X, Zhao L, Zhang S, Zhao F (2019) Study of flow characteristics of gas mixtures in a rectangular Knudsen pump. Micromachines 10(2):79. https://doi.org/10.3390/mi10020079
Publisher's Note Springer Nature remains neutral with regard to jurisdictional claims in published maps and institutional affiliations. 